\title{
High-Performance Flexible Waveguiding Photovoltaics
}

\author{
SUBJECT AREAS: \\ SOLAR ENERGY AND \\ PHOTOVOLTAIC \\ TECHNOLOGY \\ MICRO-OPTICS \\ GREEN PHOTONICS \\ SOLAR CELLS \\ Chun-Hsien Chou', Jui-Kang Chuang \& \& Fang-Chung Chen ${ }^{3}$

\begin{abstract}
'Department of Photonics and Institute of Electro-optical Engineering National Chiao Tung University, Hsinchu 30010, Taiwan, ${ }^{2}$ College of Photonics and Institute of Photonics System National Chiao Tung University, Tainan 71 150, Taiwan, ${ }^{3}$ Department of Photonics and Display Institute, National Chiao Tung University, Hsinchu 30010, Taiwan.
\end{abstract}

Received

28 May 2013

Accepted

1 July 2013

Published

22 July 2013

Correspondence and requests for materials should be addressed to F.C.C. (fcchen@mail. nctu.edu.tw)

The use of flat-plane solar concentrators is an effective approach toward collecting sunlight economically and without sun trackers. The optical concentrators are, however, usually made of rigid glass or plastics having limited flexibility, potentially restricting their applicability. In this communication, we describe flexible waveguiding photovoltaics (FWPVs) that exhibit high optical efficiencies and great mechanical flexibility. We constructed these FWPVs by integrating poly-Si solar cells, a soft polydimethylsiloxane (PDMS) waveguide, and $\mathrm{TiO}_{2}$-doped backside reflector. Optical microstructures that increase the light harvesting ability of the FWPVs can be fabricated readily, through soft lithography, on the top surface of the PDMS waveguide. Our optimized structure displayed an optical efficiency of greater than $42 \%$ and a certified power conversion efficiency (PCE) of 5.57\%, with a projected PCE as high as approximately $18 \%$. This approach might open new avenues for the harvesting of solar energy at low cost with efficient, mechanically flexible photovoltaics.

U sing photovoltaic (PV) technologies to harvest sunlight is a promising approach for generating the enormous amounts of electricity required to meet the increasing worldwide demand while minimizing environmental impact. The high cost of PV systems, however, remains a barrier for scaling their installations. To make them more economical, solar concentrators can be used to collect incident solar irradiation and focus it on highly efficient PV cells, thereby decreasing the physical area of the panels ${ }^{1-4}$. Nevertheless, conventional PV concentrators require sun trackers and/or complicated optical components, which are expensive to maintain. In addition, difficulties in the thermal management of the hot spots, insufficient harvesting of diffuse sunlight, and the complexity of the module assembly further impede this technology from becoming a practical energy resource $e^{4,5}$.

Luminescent solar concentrators (LSCs), typical stationary concentration systems that do not require solar tracking, have recently received renewed interest because of their potential for achieving high optical concentrations with a flat-panel geometry ${ }^{6,7}$. LSCs usually feature an inexpensive waveguide doped with luminescent species. Incident light is absorbed by the luminescent dyes and then re-emitted at a red-shifted wavelength; a fraction of the emission is trapped in the waveguide through total internal reflection (TIR). Subsequently, the light is guided and concentrated toward one or more smaller PV cells attached to the edges of the waveguide. Unlike conventional concentrators, LSCs concentrate both direct and diffuse light and can potentially produce a high optical concentration. Since Webber and Lambe introduced the concept in 1976, several highly efficient LSCs have been developed ${ }^{3,8-10}$. For example, Slooff $e$ t al. reported the current world-record power conversion efficiency (PCE) of $7.1 \%$ by integrating four GaAs cells into an $\mathrm{LSC}^{8}$. More recently, Desmet et al. used monocrystalline silicon solar cells, which are cheaper than $I I I-V$ cells, to fabricate LSCs with a high PCE of $4.2 \%{ }^{10}$.

A general assessment of the cost of different energy technologies- the levelized cost of energy (LCOE) ${ }^{11}$, which considers the lifetime energy production and lifetime costs associated with a system- has foreseen the balance point of grid parity in the near further. Nevertheless, the cost of fabrication and materials is not the only factor limiting the wider acceptance of PVs. In recent years, there are been increasing research efforts devoted to the development of flexible energy sources. Organic photovoltaic devices (OPVs), for example, have received much attention for not only their potential low-cost fabrication but also their unique flexibility, which offers the possibility to roll, fold, and flex the solar module in portable electronics and off-grid devices with irregular shapes. Although the efficiencies of OPVs have recently been improved substantially to over $10 \%^{12}$, this technology still suffers from reliability issues and will require further improvements in module efficiencies.

Most LSCs are made of rigid substrates, such as poly(methyl methacrylate) (PMMA) or glass, and the PV cells are attached to them using an optically clear adhesive (OCA). Although sufficiently thin PMMA sheets can be 
slightly flexible ${ }^{13}$, the mechanical properties remain poor, limiting the applicability. In this paper, we describe a new generation of flexible waveguiding photovoltaics (FWPVs) prepared using a simple molding approach for integrating poly-Si solar cells, a soft polydimethylsiloxane (PDMS) waveguide, a $\mathrm{TiO}_{2}$-doped backside reflector (BSR), and optical microstructures. The unique properties of PDMS $^{14,15}$ impart this novel architecture with a high degree of flexibility and allows microstructures to be readily fabricated through soft lithography ${ }^{16}$. These FWPVs, which can be fabricated in many shapes and sizes, can be used as convenient, flexible energy sources. Unlike traditional PV modules, which require heavy cover glasses and alumina frames, the flexible FWPVs reported herein are light and easy to install. Our optimized device exhibited a PCE of close to $6 \%$; predicted PCEs are also extremely high (ca. 18\%).

\section{Results}

FWPV design and characterization. Because they are cost-effective, we used poly-Si solar cells, which are available at the lowest price on the market, in our modules. The waveguiding concentrator was made of PDMS, a hydrophobic soft material with many attractive properties, including high transparency, excellent weather resistance, low environment hazard, high mechanical elasticity, and low $\operatorname{cost}^{15,17,18}$. PDMS has also been used commonly for fabricating microfluidic systems ${ }^{19,20}$, soft lithography ${ }^{16,21}$, wafer packaging, and PV encapsulating ${ }^{22}$. Fig. 1 presents the structure of a typical FWPV. We adopted a simple molding method to fabricate the flexible solar modules (see Methods and Supplementary Fig S1). We placed one or more Si solar cells at the edges of the mold and then poured a fixed amount of Sylgard ${ }^{\circledR}$ silicone elastomer mixture (weight ratio of base and curing agent, 10:1) into the mold. The sample was thermally cured at $100^{\circ} \mathrm{C}$ for $15 \mathrm{~min}$. After solidification, another PDMS layer incorporating $\mathrm{TiO}_{2}$ nanoparticles (NPs) was fabricated using a similar method. The embedded $\mathrm{TiO}_{2}$ NPs behaved as scattering centers and the resulting $\mathrm{PDMS} / \mathrm{TiO}_{2}$ layer served as a flexible $\mathrm{BSR}$, increasing the probability of light trapping inside the waveguide. Finally, the whole module was carefully peeled off from the mold. Varying the dimensions of the acrylic mold allowed us to readily modify the size of the FWPV. For simplicity, we name a $3.0 \times$ $3.0 \times 0.6 \mathrm{~cm}^{3}$ module incorporating one solar cell as a $3 \times 31$-cell module. Because each of the modules reported herein had a thickness of $0.6 \mathrm{~cm}$, the other modules are all named following the same rule. For example, a $5 \times 54$-cell module features four attached solar cells, with the dimensions of the substrate (including the waveguide and the BSR) being $5.0 \times 5.0 \times 0.6 \mathrm{~cm}^{3}$.

Fig. 1b displays the normalized external quantum efficiency (EQE) spectra of a poly-Si solar cell and of a $3 \times 31$-cell FWPV. Although the curve of the poly-Si cell exhibited a typical spectral response, we observed significantly decreased efficiency in the response of the FWPV in the ultraviolet (UV) spectral region. We attribute the decreased photocurrent to the strong UV absorption of the $\mathrm{TiO}_{2}$ $\mathrm{NPs}^{23}$. In fact, although the PCE was somewhat low, the UV absorption might minimize the degree of damage of the solar cells from direct UV irradiation, potentially improving the lifetime of the solar modules. Fig. 2 a presents photocurrent-voltage $(I-V)$ curves of various modules (differing in size and number of cells) under simulated AM 1.5 G illumination ( $\left.100 \mathrm{~mW} \mathrm{~cm}^{-2}\right)$; note that the FWPVs containing multiple solar cells featured the cells connected in series. Interestingly, we observed higher currents for the modules containing fewer cells. For instance, the short-circuit current $\left(I_{\mathrm{sc}}\right)$ was $42.36 \mathrm{~mA}$ for the $3 \times 31$-cell module, whereas it was $31.29 \mathrm{~mA}$

a

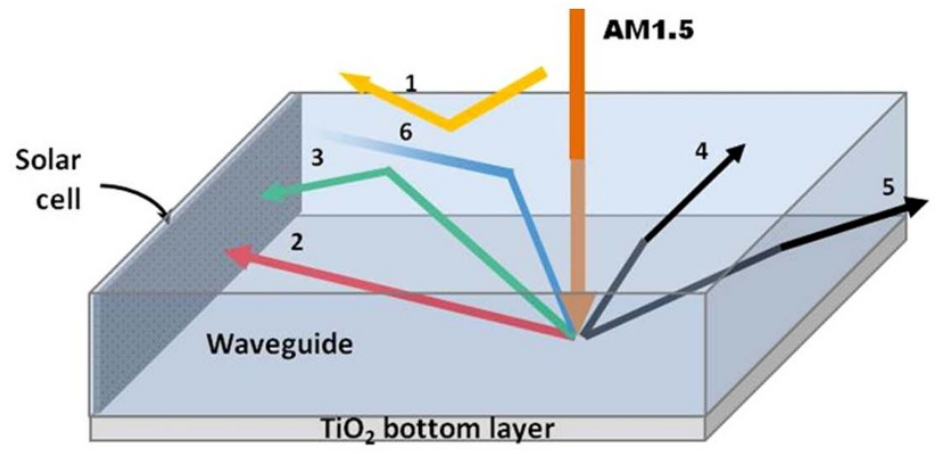

b

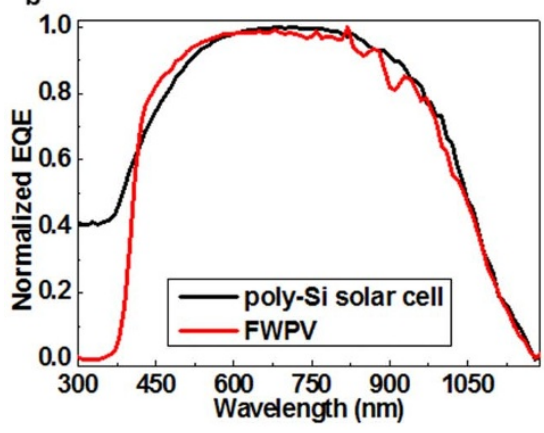

c

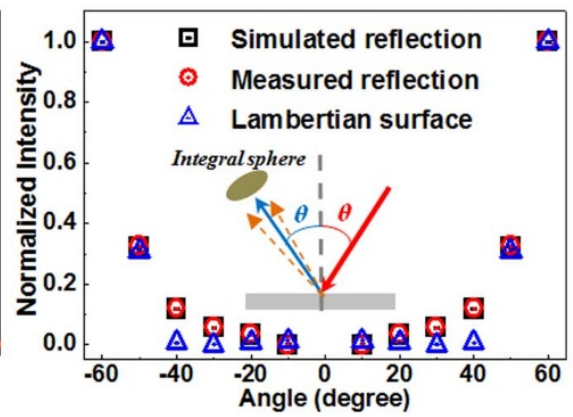

Figure 1 FWPVs. (a). Schematic three-dimensional representation and working scheme of a typical FWPV, with scattering occurring at the bottom layer. Excluding the non-trapped light (ray 1), some of the trapped light was directly incident to the solar cell (ray 2) or guided to the solar cell through TIR (ray 3), while some escaped from the top surfaces (ray 4) or edges (ray 5); furthermore, some light was absorbed by the waveguide material (ray 6). (b). Normalized EQE spectra of a poly-Si solar cell and a $3 \times 3$ 1-cell FWPV. An obvious decrease in efficiency occurred at the UV region (below ca. $360 \mathrm{~nm}$ ), due to the strong UV absorption of $\mathrm{TiO}_{2} \mathrm{NPs}$. (c). Normalized real and imitation specular reflections. Obvious differences appear at low incident angles $\left(10-40^{\circ}\right)$. The ideal Lambertian surface exhibited uniform scattering intensity at low incident angles, whereas the specular intensity of the $\mathrm{TiO}_{2}$ NP/PDMS layer increased more rapidly to become larger than that in the ideal Lambertian system. The ABg parameters were fitted to match the normalized measured results at incident angles from $10^{\circ}$ to $60^{\circ}$. 

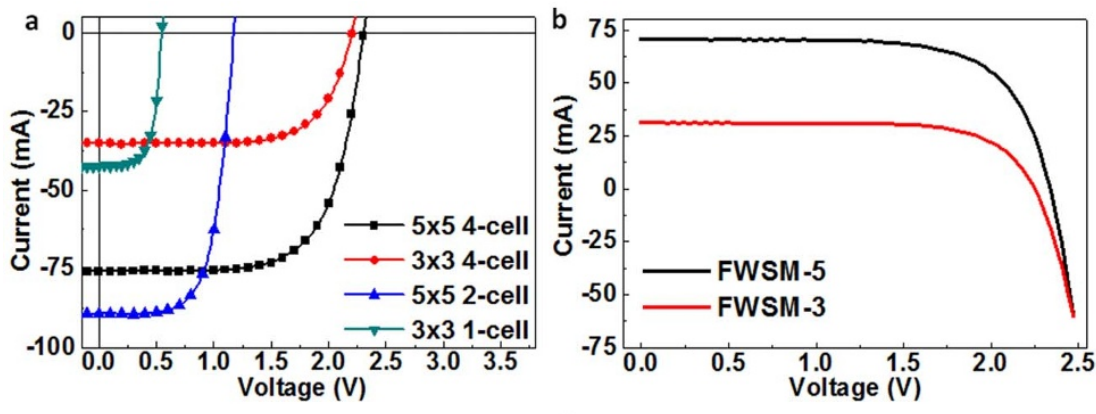

c

d
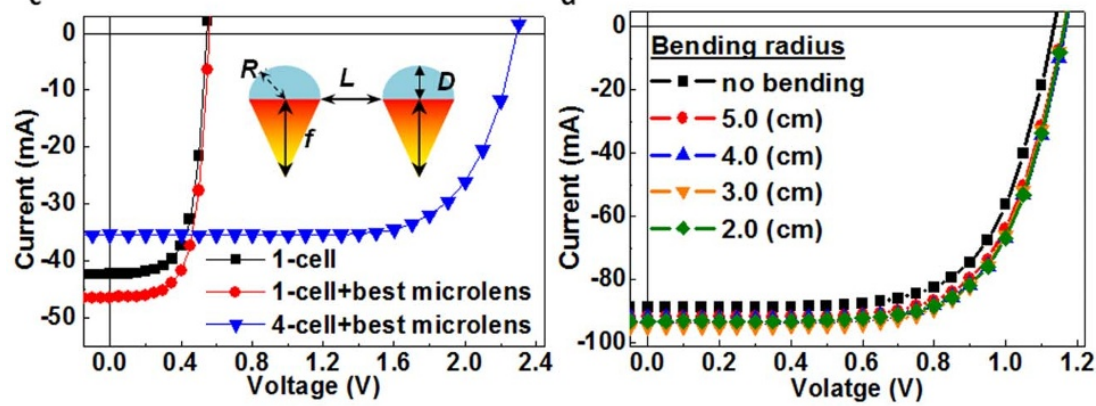

Figure $2 \mid$ Performance of the FWPVs. (a). $I-V$ characteristics of FWPVs differing in size and number of attached solar cells. (b). ITRI-certified $I-V$ data for two representative FWPVs. The $5 \times 5$ (FWSM-5) and $3 \times 3$ (FWSM-3) modules exhibited maximum powers of 115.79 and $50.09 \mathrm{~mW}$, respectively. (c). Performance of $3 \times 3$ modules prepared with and without MLs. Inset: Lens profile; the radius $(R)$, depth $(D)$, and interval $(L)$ were 0.50 , 0.76 , and $0.10 \mathrm{~mm}$, respectively. The focal length $(f)$ was calculated to be $5.0 \mathrm{~mm}$. (d). $I-V$ curves of the $5 \times 52$-cell FWPV under various bending conditions.

for the $3 \times 34$-cell module. Considering the cells were connected in series, the current is determined by the intensity of incident light absorbed by individual cells. The refractive index $(n)$ of the antireflecting coating $\left(\mathrm{SiN}_{x}\right)$ on $\mathrm{Si}$ solar cell is about 2.03 at $589 \mathrm{~nm}^{24,25}$. According to Snell's law ${ }^{26}$, in principle, the incident rays on the Si solar cells from PDMS $(n \sim 1.43)$ were not reflected. In other words, all of the photons were absorbed by the attached solar cells once they approached the surface of the cells. For 1-cell modules, the other three sides of PDMS without cell attached reflected the photons back to waveguide due to the air cladding effect $\left(\mathrm{n}_{\mathrm{air}}=1\right)$. Such additional contribution of flux resulted in higher current for the 1cell module. Further, the $3 \times 34$-cell module exhibited the highest PCE among our tested devices; the open-circuit voltage $\left(V_{\mathrm{oc}}\right)$, the value of $I_{\mathrm{sc}}$, and the fill factor (FF) were $2.26 \mathrm{~V}, 31.29 \mathrm{~mA}$, and $70.95 \%$, respectively. Table 1 summarizes the performances of all of the tested FWPVs.

One of the most important figures of merit for a concentrating system is the conversion efficiency from cell to module-also defined as the optical efficiency $\left(\eta_{o p t}\right)^{27,28}$ :

$$
\begin{aligned}
\frac{\eta_{\text {module }}}{\eta_{\text {cell }}} & =\frac{\eta_{\text {cell }} \times P_{\text {out } / 0.1}\left(W \cdot \mathrm{cm}^{-2}\right) \times A_{\text {module }}}{\eta_{\text {cell }}} \\
& =\frac{P_{\text {out }}}{0.1\left(W \cdot \mathrm{cm}^{-2}\right) \times A_{\text {module }}}=\frac{P_{\text {out }}}{P_{\text {in }}}=\eta_{\text {opt }}
\end{aligned}
$$

where $\eta_{\text {module }}$ and $\eta_{\text {cell }}$ are the PCEs of the modules and of the solar cells, respectively; $P_{\text {out }}$ and $P_{\text {in }}$ are the total output and input photon power, respectively; and $A_{\text {module }}$ is the area of the incident surface. For example, the value of $\eta_{\text {opt }}$ of the $4.2 \%$ LSC consisting of mono-Si solar cells ${ }^{10}$ is $26.9 \%$, while that of the world-record $7.1 \%$ LSC featuring GaAs cells ${ }^{8}$ is in the range $26.3-30.8 \%$. Table 1 lists the calculated values of $\eta_{\text {opt }}$ for the modules tested in this present study. The optical efficiencies were greater than $30 \%$ for our 4-cell configurations. The value of $\eta_{\text {opt }}$ of our $3 \times 34$-cell module was $44.6 \%-$ among the highest values ever reported for a flexible flat-plane concentrator system.

Two representative modules, a $3 \times 34$-cell and a $5 \times 54$-cell

\begin{tabular}{|c|c|c|c|c|c|c|}
\hline $\begin{array}{l}* 5 \times 54 \text { cell } \\
* 3 \times 34 \text { cell } \\
5 \times 54 \text { cell } \\
5 \times 52 \text { cell } \\
3 \times 34 \text { cell } \\
3 \times 31 \text { cell } \\
3 \times 31 \text { cell }+ \text { best microlens } \\
3 \times 34 \text { cell }+ \text { best microlens }\end{array}$ & $\begin{array}{l}70.71 \\
31.29 \\
71.18 \\
89.37 \\
34.42 \\
42.36 \\
46.50 \\
35.37\end{array}$ & $\begin{array}{l}2.34 \\
2.26 \\
2.29 \\
1.17 \\
2.21 \\
0.54 \\
0.55 \\
2.29\end{array}$ & $\begin{array}{l}69.85 \\
70.95 \\
67.52 \\
66.29 \\
69.58 \\
65.07 \\
66.23 \\
71.15\end{array}$ & $\begin{array}{l}4.63 \\
5.57 \\
4.40 \\
2.70 \\
5.89 \\
1.67 \\
1.90 \\
6.40\end{array}$ & $\begin{array}{l}35.07 \\
42.19 \\
33.33 \\
20.45 \\
44.62 \\
12.65 \\
14.39 \\
48.48\end{array}$ & $\begin{array}{l}4.56 \\
6.07 \\
4.56 \\
2.49 \\
6.07 \\
1.68 \\
1.86 \\
6.37\end{array}$ \\
\hline $\begin{array}{l}\text { aPCE obtained experimentally. } \\
\text { bOptical efficiency obtained experimentally. } \\
\text { cPCE predicted from the optical simulation. } \\
\text { "Values certificated by ITRI. }\end{array}$ & & & & & & \\
\hline
\end{tabular}
module, were further certified at the Photovoltaic Metrology 
Laboratory in Center for Measurement Standards of Industrial Technology Research Institute (ITRI) of Taiwan. Fig. 2b displays the results and Table 1 summarizes the data; further details are available in the Methods section and in the certification report (see Supplementary Information). The $I-V$ characteristics measured at ITRI were nearly identical to the results obtained at NCTU. The maximum power outputs of the $5 \times 54$-cell and $3 \times 34$-cell modules were $115.79 \mathrm{~mW}\left(V_{\mathrm{oc}}=2.34 \mathrm{~V} ; I_{\mathrm{sc}}=70.71 \mathrm{~mA}\right)$ and $50.09 \mathrm{~mW}$ $\left(V_{\mathrm{oc}}=2.26 \mathrm{~V} ; I_{\mathrm{sc}}=31.29 \mathrm{~mA}\right)$, respectively (Fig. $\left.2 \mathrm{~b}\right)$; their PCEs were 4.63 and $5.57 \%$, respectively, and their values of $\eta_{\text {opt }}$ were ultrahigh (35.07 and $42.19 \%$, respectively). With such high optical efficiency, we also predicted the PCEs of FWPVs featuring other types of cells attached to their edges (Supplementary Table S1). Assuming baseline efficiencies for $\mathrm{Cu}(\mathrm{In}, \mathrm{Ga}) \mathrm{Se}_{2}$ (CIGS), GaAs, and InGaP cells of 14.0, 28.0, and $37.0 \%$, respectively ${ }^{12}$, the calculated PCEs increased to $5.9,11.8$, and $15.6 \%$, respectively, suggesting a promising future for such FWPVs.

Optical simulation. To explore the optics in the FWPVs, we used a commercial ray-tracing ${ }^{29-31}$ software to simulate the experimental results. To increase the probability of trapping, mirrors, diffusers, and photonic filters are often introduced as BSRs in flat-plate concentration systems $s^{6,8,10,32-36}$. In this present study, we used a $\mathrm{PDMS} / \mathrm{TiO}_{2}$ composite layer as the backside scattering layer of the waveguide; we found that the definition of these BSRs significantly affected the accuracy of the simulation. Ideally, a $\mathrm{TiO}_{2}$ coating, as used frequently in white paintings or diffusing reflectors, would behave as a Lambertian surface ${ }^{37}$. Nevertheless, Lambertian conditions, assuming the scattering events were isotropic, did not fit the properties of the $\mathrm{TiO}_{2} \mathrm{NP}$ /PDMS layer in our FWPVs. Here, we adopted a bidirectional scatter distribution function (BSDF) by adjusting the parameters $A, B$, and $g^{38-40}$ defining the scattering properties (for details, see the Supplementary discussion and Fig. S2). As displayed in Fig. 1c, we measured the specular reflections of the PDMS/ $/ \mathrm{TiO}_{2}$ BSR at multiple angles and fitted the result to the BSDF; for comparison, we also plot an ideal Lambertian surface in the spectrum. Overall, the total integrated reflection intensity increased upon increasing the angle of incidence for both cases. Whereas the Lambertian surface exhibited a rather uniform scattering scheme at low incident angles $\left(10-40^{\circ}\right)$, the intensity of the scattering from the $\mathrm{PDMS} / \mathrm{TiO}_{2}$ layer increased rapidly upon increasing the incident angle, resulting in higher reflection intensities than those of the ideal Lambertian system. This result suggests that the specular reflection of the composite BSR might be intense relative to that of an ideal Lambertian surface at low angles of incidence. This modeling using a well-defined BSDF provided quite accurate predictions of the behavior of the FWPVs. If we consider the BSR as a Lambertian surface, the simulated efficiencies were overestimated, due to the resulting higher probability of light trapping (Supplementary Tab. S2). Further modifications for improving the scattering behavior of the $\mathrm{PDMS} / \mathrm{TiO}_{2}$ BSR are currently under investigation.

Concentrator performance. To study the concentrator performance of the FWPVs, we have calculated the optical concentration ratio $(C R)$, which is defined as the product of $\eta_{\text {opt }}$ and geometry factor $\left(C_{\mathrm{g}}\right)^{6,7}$. The $C_{\mathrm{g}}$ is the ratio of the area of concentrator aperture to the effective area of the solar cell. Take the $5 \times 54$-cell planar module as an example, its $C_{\mathrm{g}}$ and $\eta_{\mathrm{opt}}$ were 2.5 and $35.07 \%$, respectively, resulting in a $C R$ of 0.877 . Based on the simulated results ${ }^{6,7,27}$, as displayed in Fig. 3a, we predict that the 4-cell FWPV can exhibit a gainful $C R(>1)$ when the area of surface aperture is larger than $10 \times$ $10 \mathrm{~cm}^{2}$.

The concentration ratio as a function of incident angles was further investigated ${ }^{6,41}$. Figure $3 \mathrm{~b}$ presents the result and the photocurrent response of a bare $\mathrm{Si}$ solar cell is also included for comparison. We could see that the photocurrent decreased rapidly
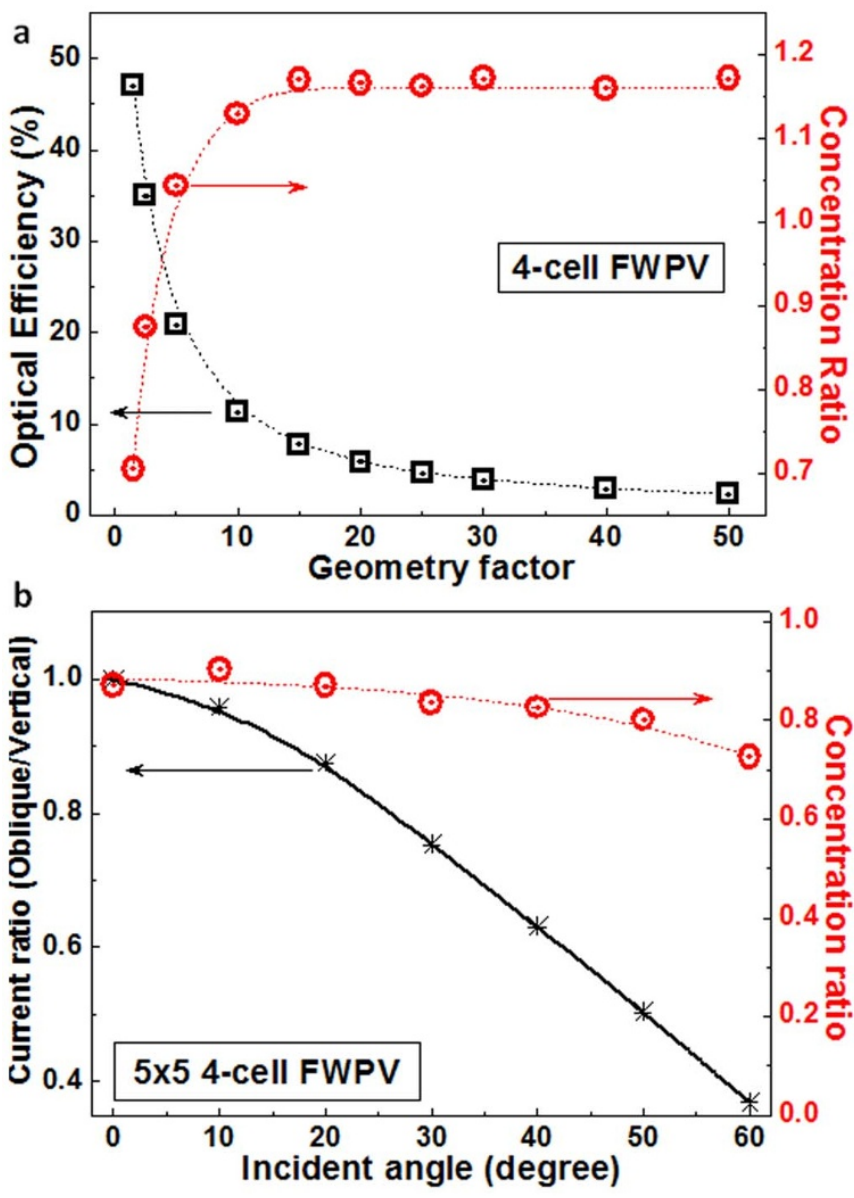

Figure $3 \mid$ Concentrator performance. (a). Optical efficiency and concentration ratio as a function of geometry factors for a 4-cell FWPV. (b). The concentration ratio of a 4-cell FWPV as a function of incidence angles. The photocurrent of a bare Si cell is also displayed for readily comparison.

with increasing incident angles. In contrast, the value of the concentration ratio almost remained the same up to entrance angle of $50^{\circ}$, suggesting the ability of FWPVs to harvest photons incident with higher angles.

Characterization of FWPVs featuring microlens arrays. For conventional LSCs, the major photon losses arise from the top surfaces and edges. Various approaches, including the use of aligned luminophors ${ }^{42}$, spectrally selective photonic structures ${ }^{34}$, and optical microstructures ${ }^{7}$, have been developed to minimize such losses. In our present systems, microstructures can readily be formed on the PDMS substrate using soft lithography ${ }^{16,21}$; therefore, we constructed a microlens array (MLA) as a representative microstructure that might increase the probability of trapping light in the waveguide ${ }^{43,44}$. We used ray-tracing simulations to design the lenses on the top surface of the PDMS waveguides; these MLAs were arranged hexagonally. We varied the focal length $(f)$ by changing the depth of the microstructure and altered the spacing between the lenses to obtain the best conditions for trapping light (Fig. S3 and Table S3). A $3 \times 3$ 1-cell module was both simulated and fabricated to confirm the results. We used an integral sphere system to measure the losses from the surfaces and edges (for the setups, see Supplementary Fig. S4); Fig. 4 presents the results. From measurements of the edge emissions, we found that the focal length affected the intensity significantly; the optimal value of $f$ was $5.0 \mathrm{~mm}$ (Fig. 4a), suggesting that, under these conditions, the 

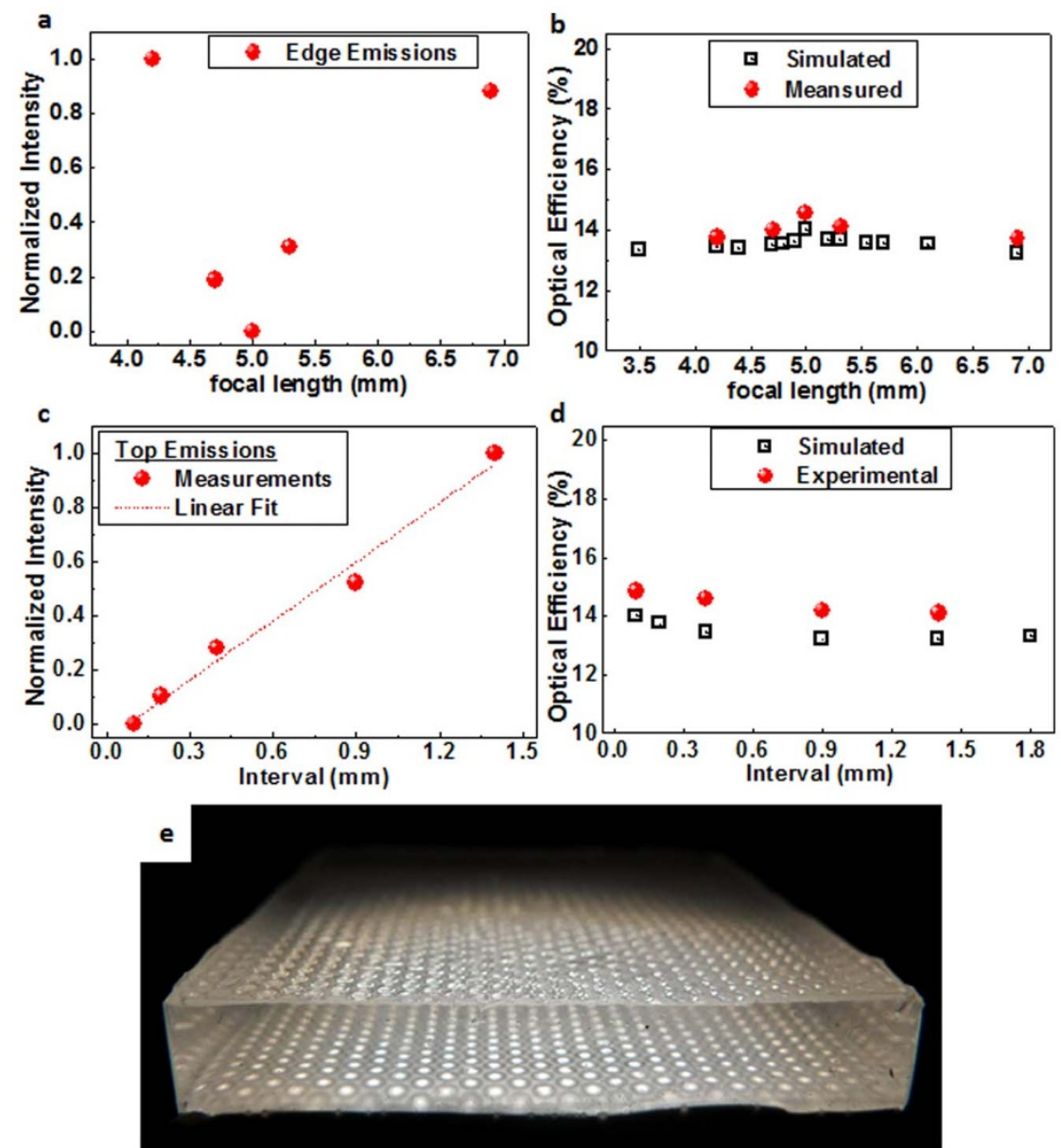

Figure $4 \mid$ Effect of lens design on the performance of a $3 \times 3$ 1-cell FWPV. (a). Dependence of lens focal length on the intensity of the edge emission. The intensity was measured using an integral sphere. The results suggested that the lowest optical loss occurred when the rays were focused on the bottom BSR. (b). Simulated and experimental optical efficiencies of FWPVs prepared with various lens designs. As a result of a lower degree of optical loss, the best-designed microstructure $(f=5.0 \mathrm{~mm})$ provided the highest value of $\eta_{o p t}$. (c.) Relationship between the intervals of the lenses and the intensity of the top surface emission. A larger interval resulted in higher probability of top losses. (d). Dependence of the lens interval on the optical efficiency. A minimal interval led to the best performance. (e). Photograph of the best-designed FWPV; the rays were clearly focused as spots on the bottom layer.

microlenses (MLs) focused the light on the BSR. The ray-tracing simulations provided consistent results (Fig. 4b); a lower degree of edge emission certainly decreased the extent of photon loss, leading to a higher optical efficiency of $13.7 \%$. The value of $\eta_{\text {opt }}$ was close to the measured value (14.39\%) obtained from the $3 \times 31$-cell module. The optical modeling further supported the notion that the bestdesigned MLA focused the light directly on the composite BSR, indicating that a larger incident angle would increase the probability of light trapping within the waveguide.

On the other hand, measurements of the top surface losses suggested that the degree of photon loss increased upon increasing the interspacing between the lenses (Fig. 4c). We infer that a high density of MLs could trap more photons reflected from the bottom surface, resulting in a higher probability of total internal reflection on the curved surfaces of the lenses. In addition, we also observed that the focal length barely affected the top loss (Supplementary Fig. S4c). Because the depth of the MLs in our design affected the value of $f$, we tested the systems using lenses having a fixed radius. Therefore, the MLs having different focal lengths actually exhibited the same curvature, resulting in the same probabilities of light escaping from the modules at various lens designs. We conclude that the curvature of the lenses, rather than the focal length, was the determination of the top surface loss.

Fig. S5 displays the $I-V$ characteristics of the $3 \times 31$-cell modules featuring various lens designs. As expected, the ray-tracing simulations and photon-loss analyses predicted the same trends for the photocurrent, further supporting the beneficial effects of the MLAs. For 1-cell modules, the optimized lens design enhanced the optical efficiency by $16.5 \%$. Fig. 2 c displays the $I-V$ characteristics of the FWPV featuring the best-designed MLA (radius, depth, and spacing of the lens array: $0.5,0.76$, and $0.10 \mathrm{~mm}$, respectively). The value of $I_{\mathrm{sc}}$ increased from 42.36 to $46.45 \mathrm{~mA}$ after incorporating the MLA, resulting in a $14.4 \%$ enhancement in PCE.

Because the cells in a 4-cell module could harvest all of the edge emissions, only the top surface loss remained in this configuration. After applying the MLA, the value of $I_{\mathrm{sc}}$ of the $3 \times 34$-cell module improved from 31.29 to $35.37 \mathrm{~mA}$ (Fig. 2c); the PCE of this module also improved by $8.6 \%$, presumably because of decreased surface loss by the MLAs. The values of $\eta_{\text {opt }}$ of such a design reached as high as $48.48 \%$. The experimental results for the 4-cell module configuration further support the fact that the microstructure on the top surface of the waveguide could decrease the degree of top surface loss. We also note that, with values of $\eta_{\text {opt }}$ as high as $48.48 \%$, the predicted PCEs of FWPVs incorporating other kinds of PV cells might also increase even further (Supplementary Table S1). For example, we calculate the PCE of an FWPV fabricated with InGaP cells to be as high as $17.9 \%$.

Mechanical flexibility of the waveguiding modules. We used bending tests to evaluate the flexibility of these FWPVs ${ }^{45}$. Although the embedded poly-Si cells were fragile, the 4 -cell modules could still 

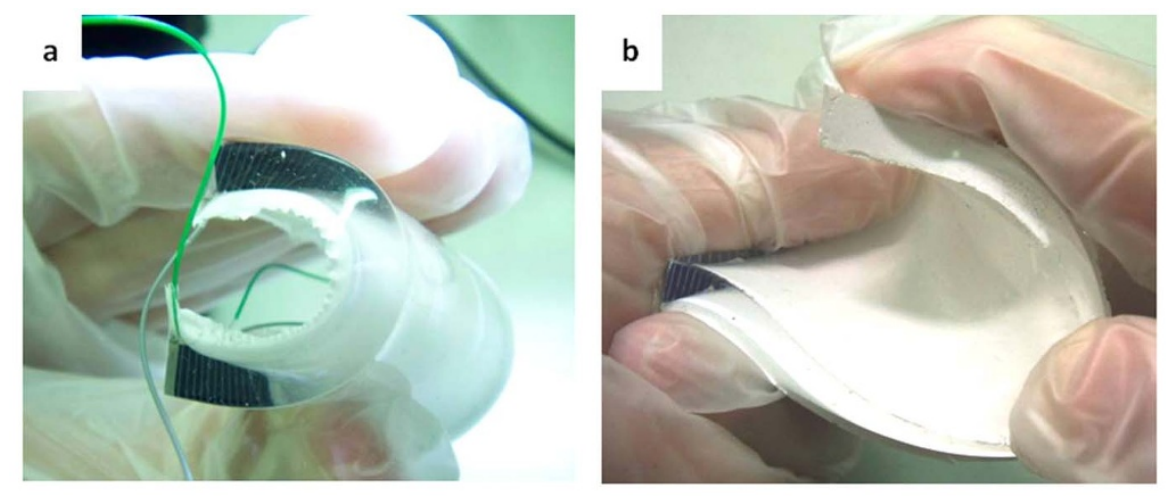

c

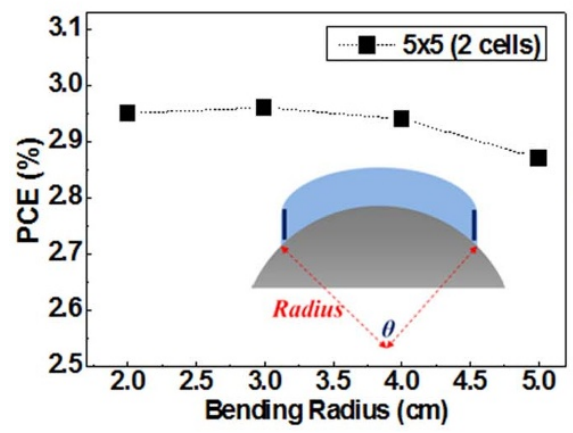

d

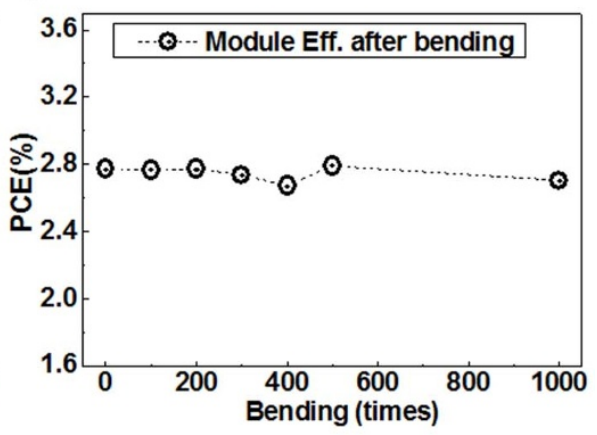

Figure $5 \mid$ Bending and stability tests of the FWPVs. (a). Photograph of a curved $5 \times 52$-cell module. The FWPV was readily bent because of its highly flexible waveguide. (b). Photograph of a twisted and bent $5 \times 51$-cell module. (c). PCEs of the $5 \times 52$-cell FWPV when bent at different radii. The efficiency increased upon decreasing the bending radius. (d). PCEs of the FWPV after various bending cycles. The FWPV maintained its efficiency almost completely after 1,000 bending cycles.

exhibit slight flexibility, especially for large-area modules; the excellent mechanical properties of PDMS somehow protected the Si cells from the bending stress. In the 2-cell configuration, the FWPVs exhibited even greater mechanically bendability. Fig. 5a reveals that a $5 \times 52$-cell module could be bent and closely attached to a curved surface. More interestingly, the 1-cell configuration exhibited an even higher degree of flexibility (Fig. 5b); it was easy to twist and its performance was barely affected after restoring the system to flatness.

We used a bending apparatus to analyze the bending effect in more detail (Supplementary Fig. S6). Fig. 5c displays the electrical performance of a $5 \times 52$-cell module at bending radii ranging from 20 to $50 \mathrm{~mm}$. Interestingly, the PCE increased upon increasing the degree of curvature (i.e., decreasing the bending radius). The PCE of the flat module was $2.71 \%$; at bending radii of $20,30,40$, and $50 \mathrm{~mm}$, it became $2.87,2.94,2.96$, and $2.95 \%$, respectively. Under such "convex" bending conditions, the cells at the edges faced the direction of the solar irradiation more directly and, therefore, received more direct flux, resulting in an improvement in efficiency of approximately $10 \%$. Furthermore, we bended the module 1000 times (to a fixed bending radius of $20 \mathrm{~mm}$ ) and measured its efficiencies. Fig. $5 \mathrm{~d}$ suggests that the module maintained almost all of its high performance even after 1000 bending cycles. The small perturbation in efficiency arose from the residue stress that remained in the waveguide during the measurements. Because the components of the FWPV, including the waveguide, back reflector, and MLs were all made of the same material (PDMS), the absence of misfit strains between different materials appears to have led to a rather stable architecture ${ }^{46}$.

\section{Discussion}

The high optical efficiencies of these FWPVs originated from several factors. In our design, the level of optical loss arising from various paths could be minimized. First of all, because the FWPVs did not incorporate any luminescent dyes, we obtained no energy loss due to absorption or emission processes and the problem of re-absorption could be avoided while the photons propagated in the waveguide. Second, integration of all of the components of the systems with PDMS waveguides eliminated several interfacial junctions, which usually lead to reflection losses. For example, OCA is often used to connect solar cells to waveguides; because the refractive index of the most common OCA (ca. 1.475) is usually different from that of the waveguide (e.g., PMMA) ${ }^{47}$, the mismatch of refractive indices would result in a significantly decreased efficiency. We have used ray-tracing simulation ${ }^{30}$ to model modules prepared with and without OCA (for simulation conditions, see Supplementary Fig. S7). The simulations indicated that the interface between the waveguide and the OCA decreased the flux to the solar cell by $15.42 \%$ (Supplementary Table S4). In other words, only $84.58 \%$ of the photons trapped in the waveguide could pass through the interface and eventually reach the solar cell. For the FWPVs reported herein, all of the trapped rays were theoretically available for absorption by the cell because of the absence of the interfaces. Moreover, in real modules, defects at the interfaces (e.g., air gaps) would also induce greater losses.

In summary, we have developed a new generation of low-cost FWPVs providing high optical efficiencies and high degrees of mechanical flexibility. In this design, optical microstructures, such as MLs, that can enhance the performance are readily integrated on the top surface of the PDMS waveguide. The lack of refractive index mismatches (that would arise from the use of OCA) and the absence of re-absorption losses (that would arise from the use of dyes) meant that we could achieve optical efficiencies as high as $48 \%$. Finally, we foresee that PCEs approaching $18 \%$ might be achievable if moreefficient solar cells ${ }^{12}$, such as $I I I-V$ photovoltaic devices, were to be integrated in these FWPVs. 


\section{Methods}

Preparation of poly-Si solar cells. The poly-Si solar cells were purchased from Tainergy Tech. The Si cells were saw-cut into small pieces and rinsed with DI water. The device area was either $3.0 \times 0.6$ or $5.0 \times 0.6 \mathrm{~cm}^{2}$, with $0.5 \mathrm{~cm}$ remaining for the light-absorbing area and $0.1 \mathrm{~cm}$ for soldering the electrode (Supplementary Fig. S1b). The poly-Si cells exhibited a PCE of $13.2 \pm 0.3 \%$; the values of $J_{s c}$ and $V_{\text {oc }}$ were $41.27 \mathrm{~mA} / \mathrm{cm}^{2}$ and $0.50 \mathrm{~V}$, respectively (Supplementary Fig. S1c). The lower efficiency presumably arose from damage during the die-saw processes and/or wire soldering.

Fabrication of FWPVs. An integral molding method was used to fabricate the flexible solar modules (Supplementary Fig. S1a). Solid acrylic molds were adopted to fix the volumes and shapes of the modules. For example, $3.1 \times 3.1 \times 1 \mathrm{~cm}^{3}$ square molds, with gaps of $0.1 \mathrm{~cm}$ left for positioning the $S i$ solar cells at the side walls, were used for prepare the $3.0 \times 3.0 \times 0.6 \mathrm{~cm}^{3}$ modules. The PDMS polymer and the curing agent (Sylgard ${ }^{\circledR}$ 184, Dow Corning) were mixed well at a weight ratio of $10: 1$; the mixture was then degassed using a vacuum pump. After positioning the Si cells in the mold, a fixed quantity of the PDMS mixture was then transvased into the mold. The mold was then thermally cured at $120^{\circ} \mathrm{C}$ for $15 \mathrm{~min}$. The thickness of the resulting flexible waveguide was $0.50 \mathrm{~cm}$. The diffusing backside reflective layer was prepared using a similar process. $\mathrm{TiO}_{2}$ (PT-501R, Ishihara Sangyo Kaisha) having a particle radius of $0.2 \mu \mathrm{m}$ was mixed with the PDMS pre-polymer. The concentration of the $\mathrm{TiO}_{2} \mathrm{NP}$ was $0.044 \mathrm{~g} / \mathrm{mL}$. After degassing, the white mixture was transvased into the mold (on the cured waveguiding layer) and then heated at $120^{\circ} \mathrm{C}$ for $15 \mathrm{~min}$. After solidification, the thickness of the BSR was $0.10 \mathrm{~cm}$. Finally, the sample was carefully peeled from the acrylic mold. The thickness of each layer could be controlled by varying the volume of the PDMS pre-polymer. For fabrication of MLAs, designed glass masks (Supplementary Fig. S3) were placed at the bottom of the molds. Using conventional soft lithography ${ }^{21}$, MLs were superimposed on the top surfaces of the PDMS waveguides.

Device characterization. The device area was determined using an optical microscope. All $I-V$ measurements were first tested at NCTU under AM1.5 G illumination at $100 \mathrm{~mW} / \mathrm{cm}^{2}$ using a $300-\mathrm{W}$ class-A solar simulator (SAN-EI Electric); the light intensity was calibrated by a standard Si diode (Hamamatsu). The EQE spectra were obtained using an EQE measurement system (Enli Technology) comprising a quartz-tungsten-halogen lamp, a monochromator, an optical chopper, a lock-in amplifier, and a calibrated Si-based diode.

Module certification. Representative $3 \times 34$-cell and $5 \times 54$-cell FWPVs were certificated by the Photovoltaic Metrology Laboratory at the Center for Measurement Standards of ITRI, certified (IEC 61215 CBTL) by the International Electrotechnical Commission System for Conformity Testing and Certification of Electrotechnical Equipment and Components (IECEE). The certification report is provided in the Supplementary Information.

Optical simulation. A commercial ray-tracing software, TracePro ${ }^{\circledR}$ (Lambda Research), was used to simulate the optics in the FWPVs. Imitation light, with wavelengths ranging from 305 to $1350 \mathrm{~nm}$, according to IEC 60904-9 (Supplementary Fig. S8a), was introduced with respect to the normal of the waveguides. The absorption of PDMS was measured using a UV-VIS spectrometer (PerkinElmer); the measured absorbencies and referred refractive indexes at 486,589 , and $651 \mathrm{~nm}$ were built into the software to calculate the light extinction coefficients (Supplementary Fig. S8b). The specular reflections under multiple angles were recorded using a spectrophotometer (U-4100, Hitachi High-Tech) comprising a 50W halogen lamp, a monochromator, a variable-angle absolute reflectance accessory, and an integrating sphere. Prior to measurement, the monochromatic light intensity at each angle was calibrated with mirrors relocated to specified positions (from 10 to $60^{\circ}$ ); the light intensity of the reflectance was collected using the integrating sphere (inset to Fig. 1c).

1. Weber, W. H. \& Lambe, J. Luminescent greenhouse collector for solar radiation. Appl. Opt. 15, 2 (1976).

2. Debije, M. G. \& Verbunt, P. P. C. Thirty Years of Luminescent Solar Concentrator Research: Solar Energy for the Built Environment. Adv. Energy Mater. 2, 12-35 (2012)

3. Currie, M. J., Mapel, J. K., Heidel, T. D., Goffri, S. \& Baldo, M. A. High-Efficiency Organic Solar Concentrators for Photovoltaics. Science 321, 226-228 (2008).

4. Yoon, J.et al. Flexible concentrator photovoltaics based on microscale silicon solar cells embedded in luminescent waveguides. Nat Commun 2, 343 (2011).

5. Nguyen, D. D., Lehman, B. \& Kamarthi, S. in Energy Conversion Congress and Exposition, 2009. IEEE. 3357-3362.

6. Kim, J. M. \& Dutta, P. S. Optical efficiency-concentration ratio trade-off for a flat panel photovoltaic system with diffuser type concentrator. Sol. Energy Mater. \& Sol. Cells 103, 35-40 (2012).

7. Karp, J. H., Tremblay, E. J. \& Ford, J. E. Planar micro-optic solar concentrator. Opt. Express 18, 1122-1133 (2010)

8. Slooff, L. H. et al. A luminescent solar concentrator with $7.1 \%$ power conversion efficiency. Phys. Status Solidi - R 2, 257-259 (2008).
9. Verbunt, P. P. C. et al. Increased efficiency of luminescent solar concentrators after application of organic wavelength selective mirrors. Opt. Express 20, A655-A668 (2012).

10. Desmet, L., Ras, A. J. M., de Boer, D. K. G. \& Debije, M. G. Monocrystalline silicon photovoltaic luminescent solar concentrator with $4.2 \%$ power conversion efficiency. Opt. Lett. 37, 3087-3089 (2012).

11. Darling, S. B., You, F., Veselka, T. \& Velosa, A. Assumptions and the levelized cost of energy for photovoltaics. Energy Environ. Sci. 4, 3133-3139 (2011).

12. Green, M. A., Emery, K., Hishikawa, Y., Warta, W. \& Dunlop, E. D. Solar cell efficiency tables (version 41). Prog. Photovolt: Res. Appl. 21, 1-11 (2013).

13. Lunstroot, K. et al. Ionic liquid as plasticizer for europium(iii)-doped luminescent poly(methyl methacrylate) films. Phys. Chem. Chem. Phys. 12, 1879-1885 (2010).

14. Mata, A., Fleischman, A. \& Roy, S. Characterization of Polydimethylsiloxane (PDMS) Properties for Biomedical Micro/Nanosystems. Biomed. Microdevices 7, 281-293 (2005).

15. Mukhopadhyay, R. When PDMS isn't the best. Anal. Chem. 79, 3248-3253 (2007).

16. Michel, B. et al. Printing meets lithography: Soft approaches to high-resolution patterning. IBM J. Res. Develop. 45, 697-719 (2001).

17. Owen, M. J. Why silicones behave funny. ChemTech 11, 5 (1981).

18. Cai, D. K. \& Neyer, A. Cost-effective waveguide integration method for large-scale electrical-opticalcircuit-board production. Electron. Lett. 46, 581-583 (2010).

19. Lee, J. N., Park, C. \& Whitesides, G. M. Solvent Compatibility of Poly(dimethylsiloxane)-Based Microfluidic Devices. Anal. Chem. 75, 6544-6554 (2003).

20. Zhang, M., Wu, J., Wang, L., Xiao, K. \& Wen, W. A simple method for fabricating multi-layer PDMS structures for 3D microfluidic chips. Lab on a Chip 10, 1199-1203 (2010).

21. Chang-Yen, D. A., Eich, R. K. \& Gale, B. K. A monolithic PDMS waveguide system fabricated using soft-lithography techniques. J. Lightwave Technol. 23, 2088-2093 (2005).

22. Kempe, M. in Photovoltaic Specialists Conference (PVSC), 2011 37th IEEE. 000085-000090.

23. Yang, H., Zhu, S. \& Pan, N. Studying the mechanisms of titanium dioxide as ultraviolet-blocking additive for films and fabrics by an improved scheme. J. Appl. Polym. Sci. 92, 3201-3210 (2004).

24. Sopori, B. Silicon nitride processing for control of optical and electronic properties of silicon solar cells. J. Electron. Mater. 32, 1034-1042 (2003).

25. Kang, M. H., Ryu, K., Upadhyaya, A. \& Rohatgi, A. Optimization of SiN AR coating for Si solar cells and modules through quantitative assessment of optical and efficiency loss mechanism. Prog. Photovolt: Res. Appl. 19, 983-990 (2011).

26. Hecht, E. Optics. 4th edn (Addison Wesley, 2002).

27. Roncali, J. \& Garnier, F. Photon-transport properties of luminescent solar concentrators: analysis and optimization. Appl. Opt. 23, 2809-2817 (1984).

28. Wilson, L. R. Luminescent Solar Concentrators: A Study of Optical Properties, Reabsorption and Device Optimisation Ph.D. thesis, Heriot-Watt University (2010).

29. Richards, B. S. \& McIntosh, K. R. in 21st European Photovoltaic Solar Energy Conference 4 (Dresden, Germany, 2006).

30. Meyer, T. J. J., Hlavaty, J., Smith, L., Freniere, E. R. \& Markvart, T. Ray racing techniques applied to the modelling of fluorescent solar collectors. Proc. of SPIE 7211, 72110N-72110N (2009).

31. Hernandez-Noyola, H., Potterveld, D. H., Holt, R. J. \& Darling, S. B. Optimizing luminescent solar concentrator design. Energy Environ. Sci. 5, 5798-5802 (2012).

32. Farrell, D. J. \& Yoshida, M. Operating regimes for second generation luminescent solar concentrators. Prog. Photovoltaic: Res. Appl. 20, 93-99 (2012).

33. Goldschmidt, J. C. et al. Increasing the efficiency of fluorescent concentrator systems. Sol. Energy Mater. \& Sol. Cells 93, 176-182 (2009).

34. Debije, M. G. et al. Effect on the output of a luminescent solar concentrator on application of organic wavelength-selective mirrors. Appl. Opt. 49, 745-751 (2010).

35. Rau, U. \& Glaeser, G. C. in Photovoltaic Energy Conversion, Conference Record of the 2006 IEEE 4th World Conference. 205-208.

36. Peters, M. et al. Spectrally-Selective Photonic Structures for PV Applications. Energies 3, 171-193 (2010).

37. Bahadur, B. in Liquid crystals- Applications and Uses Vol. 2 (ed Birendra Bahadur) Ch. 8, 397 (World Scientific, 1991).

38. Jonsson, J. C., Lee, E. S. \& Rubin, M. Light-scattering properties of a woven shadescreen material used for daylighting and solar heat-gain control. Proc. of SPIE 7065, 70650R-70650R (2008).

39. Zheng, Z. R., Zhou, J. \& Gu, P. Roughness characterization of well-polished surfaces by measurements of light scattering distribution. Optica Applicata XL, 8 (2010).

40. Pfisterer, R. N. Approximated scatter models for stray light analysis. Optics \& Photonics News 22, 2 (2011).

41. Smestad, G., Ries, H., Winston, R. \& Yablonovitch, E. The thermodynamic limits of light concentrators. Solar Energy Materials 21, 99-111 (1990).

42. Mulder, C. L. et al. Dye alignment in luminescent solar concentrators: I. Vertical alignment for improved waveguide coupling. Opt. Express 18, A79-A90 (2010).

43. Wang, C., Hirst, L. S. \& Winston, R. Optical design and efficiency improvement for organic luminescent solar concentrators. Proc. of SPIE 8124, 81240O-81240O (2011).

44. Davies, P. A. Light-trapping lenses for solar cells. Appl. Opt. 31, 6021-6026 (1992). 
45. Han, T.-H. et al. Extremely efficient flexible organic light-emitting diodes with modified graphene anode. Nat Photon 6, 105-110 (2012).

46. Suo, Z., Ma, E. Y., Gleskova, H. \& Wagner, S. Mechanics of rollable and foldable film-on-foil electronics. Appl. Phys. Lett. 74, 1177-1179 (1999).

47. Kastelijn, M. J., Bastiaansen, C. W. M. \& Debije, M. G. Influence of waveguide material on light emission in luminescent solar concentrators. Opt. Mater. 31 , $1720-1722$ (2009)

\section{Acknowledgements}

We thank the National Science Council of Taiwan and the Ministry of Education of Taiwan (through the ATU program) for financial support.

\section{Author contributions}

C.H.C. and F.C.C. conceptualized the work. C.H.C. and J.K.C. contributed to module fabrication and optical modeling. C.H.C. and F.C.C. prepared the manuscript. F.C.C. planned and supervised the project. All authors discussed the results and commented on the manuscript.

\section{Additional information}

Supplementary information accompanies this paper at http://www.nature.com/ scientificreports

Competing financial interests: The authors declare no competing financial interests.

How to cite this article: Chou, C., Chuang, J. \& Chen, F. High-Performance Flexible Waveguiding Photovoltaics. Sci. Rep. 3, 2244; DOI:10.1038/srep02244 (2013)

(c) (i) $\Theta$ This work is licensed under a Creative Commons Attribution-

By nc No NonCommercial-NoDerivs 3.0 Unported license. To view a copy of this license, visit http://creativecommons.org/licenses/by-nc-nd/3.0 